\title{
Análisis del consentimiento informado en pacientes con cáncer: una propuesta de algoritmo decisional
}

\section{Analysis of informed consent in cancer patients: a proposal for a decisional algorithm}

\author{
José Enrique Gómez Álvarez, * \\ Nora Hilda Chávez Hernández**
}

https://doi.org/10.36105/mye.2021v32n2.05

\section{Resumen}

Una de las dificultades para la atención de los pacientes oncológicos es la posibilidad de que no colaboren con su tratamiento. A partir del análisis conceptual y del estudio de dos casos, se propone mejorar la calidad del procedimiento en el consultorio de oncología de atención ambulatoria en la Clínica 180 del IMSS. ${ }^{1}$ La literatura clínica revela diversas limitaciones de los pacientes al momento de consentir tratamientos; una muy frecuente es la mención del estrés. En los casos analizados aparecen, además, informaciones previas equivocadas sobre el tratamiento y la gravedad de la enfermedad. A partir de los datos encontrados, se diseñó un algoritmo decisional para señalar los momentos que pueden facilitar y permitir el consentimiento informado. Se con-

\footnotetext{
* Doctor en Filosofía por la Universidad de Navarra. Maestro en Gerontología Social. Profesor e investigador del CISAV. México. Correo electrónico: jegomezalvarez@ yahoo.com https://orcid.org/0000-0002-8964-2207

** Oncóloga médica. Unidad de Medicina de Atención Ambulatoria en la Clínica 180 del Instituto Mexicano del Seguro Social, Valle de Chalco, Estado de México. Correo electrónico: hchavezc@unmsm.edu.pe https://orcid.org/0000-0002-9247-5227 Recepción: 10 de diciembre de 2020. Aceptación: 20 de enero de 2021.
} 
cluye que el consentimiento debe darse a lo largo de todo el proceso, y que el algoritmo decisional es una propuesta de mejora en este sentido.

Palabras clave: estrés, acompañamiento, duelo.

\section{Introducción}

Una de las dificultades en la atención de los pacientes de cáncer es la posibilidad de que no colaboren con su tratamiento. Intuitivamente queda claro que la persona tiene el derecho a no tratarse, si así lo considera, conforme con el principio de autonomía. La pregunta que se plantea en este artículo es: ¿Cómo cerciorarse en estos casos de que se ha dado un efectivo consentimiento informado? Se discuten dos ejemplos de casos clínicos, en los que no quedan del todo claras las razones del rechazo al tratamiento, pero que dan las pistas o claves de cómo deben manejarse estas situaciones. Orientar al paciente en las opciones de tratamiento y las consecuencias de tratarse o no, implica delimitar adecuadamente lo que debe esperarse del consentimiento informado. Así, a partir de los casos y de la revisión de la literatura clínica al respecto, se propone una mejora de la atención ${ }^{2}$ en el consultorio de oncología de atención ambulatoria en la Clínica 180 del IMSS.

\section{Desarrollo}

Uno de los factores que limitan la capacidad de aceptar o no tratamientos $-\mathrm{O}$, a veces, el solo vislumbrar posibilidades- es el estrés al que están sometidos los pacientes. De hecho: ... aproximadamente cuatro de cada diez pacientes de cáncer muestran niveles de distrés clínico en algún momento del proceso de la enfermedad. Esta respuesta emocional parece estar modulada por distintos factores, entre los que se encuentran aquellos relacionados con la enfermedad (1). 
En los cuidados paliativos domiciliarios relacionados con pacientes con cáncer, el « $90 \%$ de los familiares y cuidadores primarios destacó la importancia de la disminución del estrés, ante la preparación previa al ingreso hospitalario hecha por el equipo multidisciplinario de cuidados paliativos» (2). De nuevo, los aspectos emotivos afectan la percepción de la información y la colaboración. De acuerdo con la terapia cognitiva-conductual, los sentimientos ansiosos esconden o están asociados a pensamientos automáticos, que suelen generar conductas de seguridad.

Un enfoque ya clásico en el campo de la bioética clínica es la utilización de los cuatro principios: beneficencia, no maleficencia, autonomía y justicia (3). Dentro de éstos, la autonomía se reconoce como el principio rector en el caso que abordamos aquí, porque ese principio entra en conflicto con el de beneficencia. Si los pacientes deciden no atenderse y no parece haber razones clínicas proporcionadas, entonces, ¿qué debemos o podemos hacer para que en un momento dado reconsideren su decisión sin atentar contra su autonomía?

Probablemente, una de las claves es el concepto de comunicación. Proporcionar información clara y concisa sobre el cáncer, permitiendo la expresión de pensamientos y sentimientos sobre el estado actual del trastorno, las expectativas y emociones asociadas, y confrontar lo anterior con la realidad, al mismo tiempo que se valora el papel del posible apoyo o rechazo de la familia y amigos, son cruciales para la posibilidad de afrontar la enfermedad $(4,5)$. En otras palabras, se trata de brindar una auténtica consejería y no sólo proporcionar información (4). Otro concepto esencial es la confianza. Si no se busca una estrategia de comunicación en un clima de confianza (A), se torna casi imposible evaluar la situación del paciente en cuanto a su capacidad de razonar y de medir los riesgos y beneficios de su propia decisión (4). Dicho de otro modo, un consentimiento informado adecuado implica la certeza, por parte del paciente, de que sus decisiones serán respetadas y, por otro lado, de que la información proporcionada pueda ser 
cuestionada y discutida en el mismo proceso sin sanciones morales o sociales para el paciente. Estas sanciones van desde la expresión del desagrado del médico tratante hasta que éste muestre impaciencia ante las preguntas «ingenuas» del paciente o, incluso, ante las amenazas de retiro del tratamiento. Estos elementos que hemos subrayado deben ser incluidos en el mismo proceso decisional (B). En los distintos momentos de contacto con los pacientes (véase Figura 1), hay otros factores que hay que considerar en el proceso del consentimiento como son: el nivel cultural, la edad, el tipo de tumor, el pronóstico, la agresividad del tratamiento, la necesidad de una cirugía agresiva y potencialmente curativa, la actitud del médico, la educación sanitaria del médico, el bagaje cultural, la experiencia previa, la psicología, el entorno, las expectativas y los prejuicios, entre otros (C) (5). Todos estos factores hay que considerarlos como parte del proceso del consentimiento realmente individualizado, aunque la hoja escrita que suela utilizarse para efectos sea la misma para todos los pacientes. Nos encontramos, pues, con el problema de la verdad tolerable para el paciente (D). Su «cálculo» es prudencial, en el sentido de que debe medirse aquí y ahora cuánta información puede manejar y asimilar el paciente para tomar decisiones sobre su vida. Ese cálculo prudencial debe verse desde dos ángulos: el del personal de salud y el del paciente. El personal de salud debe medir justamente su capacidad de emitir o evaluar una intervención médica en su justo balance de costo-beneficio. Ese balance no se da sólo por el talento médico: implica un juicio, en el que ese talento encuentre un justo medio dictado por la razón en el caso concreto. Así, un médico con tendencia a la generosidad bien puede perderse en su juicio respecto de su paciente, dándole esperanzas infundadas de mejoría debido a su tendencia optimista. El optimismo debe regularse por la prudencia para dar en el blanco de la virtud de la gentileza/optimismo, que es un punto medio entre la aridez del pesimismo y el optimismo inmoderado. 
El balance señalado también aplica al paciente: uno con demasiado optimismo puede aceptar, por ejemplo, tratamientos experimentales que no tienen proporción clínica alguna. Por ejemplo, un paciente con cáncer gástrico, etapa IV, metastásico con ECOG 3, (un estado funcional en el que se mantiene en cama más de la mitad del día, habiendo tenido tres líneas de tratamiento paliativo según el progreso de la enfermedad), pudiera recibir y aceptar la oferta de algún tratamiento como la temozolomida. Sin embargo, en ese caso, lo más probable es que esa terapia cause daño físico al paciente, sin proporcionarle algún beneficio relevante.

La prudencia garantiza de algún modo la consecución de la virtud pero, de una manera circular, la virtud, a su vez, permite la correcta orientación a la prudencia. En otras palabras, si no regulamos nuestras tendencias al placer y la aversión al dolor se sesga la prudencia. ¿Qué hacemos entonces? ¿Qué regulamos primero? La prudencia como virtud intelectual nos permite «medin» si la norma es aplicable aquí y ahora. Para el médico, preguntas como si el protocolo es adecuado a las circunstancias del paciente, son indispensables en casi cualquier decisión clínica. Al mismo tiempo, el paciente, por analogía, se cuestiona si tiene fuerza física y disposición para afrontar el tratamiento. La prudencia, pues, no es «astucia» para buscar los medios adecuados a mis intereses, sino la búsqueda de los medios adecuados a la virtud (6). Pero, ¿no es, acaso, un argumento circular? Resulta que hay que aprender a ser virtuoso para ser prudentes, pero se debe ser prudente para dar con la virtud. La respuesta es que la virtud se aprende junto con el juicio. Así, cuando queremos ser temperantes, lo hacemos evaluando nuestras tendencias y emitiendo un juicio acerca de ellas. No se aprende por puro condicionamiento, sino que va aparejada una evaluación racional de la situación (aunque el juicio se equivoque). Cómo resume Vigo: no se puede identificar en abstracto el contenido de la prudencia sin indicar cómo actúa el prudente frente a cada cosa. Y, viceversa, el virtuoso no puede ser identificado como tal sin hacer referencia a la prudencia; es decir, el 
valiente es el prudente frente al miedo. Hay un ir y volver entre virtud moral y prudencia (6).

El paciente oncológico no se vuelve valiente al razonar su situación, sino al enfrentarse a la situación evaluando riesgos, beneficios y la adecuación para su desarrollo personal. Esa evaluación se hace con el apoyo del personal de salud y no al margen de éste. La propuesta de mejora de la atención en el consentimiento informado no es sólo una actitud intelectualista: el personal de salud no sólo informa y proporciona datos de los procedimientos, sino que ayuda al paciente a confrontarlos evaluando desde la perspectiva de las virtudes. Lo anterior, por supuesto, supone más tiempo que el llenar un mero formato o pedir una firma. Implica dedicación y un ajuste a los tiempos de atención que suelen ser, lamentablemente, muy cortos en tiempo en el sector público de salud (E).

Pues bien, estos elementos señalados deben considerarse en el proceso de consentimiento informado: buscar la virtud con el juicio proporcionado al ofrecer alternativas de salud y al recibirlo, por lo que:

No es recomendable que en los primeros momentos se pretendan atar todos los cabos y dar por terminada la labor de información y de relación con el enfermo. En la primera entrevista se corre el riesgo de que cualquier información que se dé sea demasiada. Además, el médico no tiene aún referencias para conocer qué entiende o qué extrae el enfermo del aluvión de datos que se le proporciona. No es preciso pretender suministrar toda la información en una sola conversación: es preciso contar con el factor tiempo para que el enfermo y la familia maduren los datos que se les aportan.

El médico, a su vez, sufre una dualidad: por un lado, su conocimiento es técnico (poiesis) y debe buscar el medio más claro para eliminar/ disminuir un tumor canceroso; su racionalidad técnica parece no implicar la perfección de sí mismo. Y, por otro lado, debido a que el cáncer no es una entidad separada de la realidad personal del enfermo, también su acción debe enfocarse a sí mismo. Por ejem- 
plo, cuando se atiende a un paciente, también se realiza por un bien virtuoso del médico o del personal de salud en general, y se cuida al paciente por la virtud de la generosidad. Así, las actividades y procesos clínicos son duales: se busca un fin extrínseco y otro intrínseco. En otras palabras: no sólo se debe ser buen médico/enfermero(a)/trabajador social, sino una buena persona, médico, enfermero, etcétera. Esta dualidad se debe considerar en los procesos de atención del paciente: equilibrar la atención sin descuidarse a uno mismo (F). Lo anterior implica el proceso de consentimiento.

Un mal personal de salud produce o puede producir resultados técnicos malos, al desviarse del objetivo de hacerse bien y hacer bien al otro desde el punto de vista ético. El médico puede prescribir una terapia conforme con el protocolo porque sabe que sirve, pero lo hace con el paciente equivocado, con el que, por ejemplo, no podría cumplir con la terapia. Ahí la técnica ha quedado desvirtuada. Vigo lo resume muy bien: ...la técnica es moralmente neutral, pero en el sentido de que por medios meramente técnicos no es posible determinar para qué fines se utilizan los instrumentos que la técnica pone a disposición. En definitiva, los fines para los que se aplican los instrumentos técnicos parecen deseables por consideraciones no de tipo técnico, sino moral (6).

Más adelante, a partir de las categorías propuestas se presenta un algoritmo decisional para pacientes con diagnóstico de cáncer que considere la experiencia en la práctica clínica, que ayude a disminuir las reticencias y a garantizar, dentro de lo posible, un auténtico consentimiento por parte del paciente, buscando el bien intrínseco tanto del personal de salud como de los pacientes. De esa manera, en el mismo se señalan áreas que implican esa dualidad virtud/prudencia del personal de salud y del médico, y que se resaltan en el presente texto.

Para ejemplificar la necesidad de dicho algoritmo, a continuación revisamos dos casos verídicos de pacientes que hemos constatado en nuestra práctica clínica, para posteriormente pasar a la propuesta. 
Caso 1

Mujer de 52 años. Hipertensa en tratamiento con enalapril. Se autodetectó masa de más de $2 \mathrm{~cm}$ en mama izquierda. Mastografía con datos de probable malignidad. Biopsia con aguja de corte con datos de malignidad. Cáncer ductal de mama izquierda, etapa clínica IIIA. Receptores de estrógeno de más de $100 \mathrm{fmol}$; receptores de progesterona $90 \%$; HER2NEU negativo. Se envió a oncocirugía para mastectomía, pero la paciente no aceptó. En su caso, la cirugía la podría haber dejado sin tumor y tendría probabilidad incluso de quedar sin cáncer de por vida.

Se negó a la mastectomía, porque «ha visto personas que las operan y vuelven a lo mismo». La paciente cree que la intervención no es efectiva.

Aquí encontramos el caso de una paciente que ha rechazado el tratamiento, por una probable valoración incorrecta del costo-beneficio y probablemente también por falta de una información detallada y, podríamos decir, visualizada, del proceso al que debe someterse. Uno de los temores de los pacientes es tener que soportar procesos desconocidos. En ese sentido no sólo es útil, sino necesario ilustrar los procedimientos, como, por ejemplo, lo hace el servicio de cuidados paliativos del Instituto Nacional de Cancerología (3). Otra estrategia es que el paciente pueda visitar y ver a otros pacientes en sus mismas circunstancias de tratamiento, para disminuir el temor ante ese mismo proceso (G). Naturalmente, ello implica un proceso de seguimiento que absorbe buena parte del tiempo disponible y que en ocasiones es prácticamente imposible (4); sin embargo, pensamos que ésa es una de las estrategias que, sin manipular al paciente, le permite tomar una verdadera decisión informada. Ese proceso de familiarización con el entorno puede $y$, a nuestro juicio, debe ser realizado por el personal de enfermería que, a menudo, es el que acompaña en muchos momentos al paciente durante los tratamientos.

Una segunda estrategia que nos sugiere este caso es valorar si el consentimiento está o no realmente fundado en la propia experiencia y en las expectativas del paciente $(\mathrm{H})$. En ocasiones, los pacientes toman la decisión influidos por el entorno familiar o, por el 
contrario, se teme que, al aceptar el tratamiento, después no sea posible retractarse de su decisión, a veces por la propia presión de la familia $(2,5)$. Una de las posibles estrategias es utilizar el test minimental ${ }^{3}$ como criterio de la capacidad de decisión del paciente. Con esto tendríamos varios criterios para perfeccionar el consentimiento informado del paciente. Veamos ahora el segundo caso.

Caso 2

Mujer de 59 años. Refiere alergia a penicilina y cortisona. Hipertensión en tratamiento homeopático. Se palpó masa en mama izquierda en 2015. No acudió al médico inicialmente, por miedo. En febrero de 2018 se agregó ulceración; fue entonces con un médico particular. 25 abril de 2018: biopsia de tumoración de mama izquierda con reporte histopatológico el 30 abril de 2018: carcinoma ductal infiltrante sin patrón específico, Grado III histológico de la clasificación Scarff Bloom Richardson, con permeación linfática y extensas zonas de necrosis. Fue valorada por oncocirugía el 14 mayo de 2018, no siendo candidata a tratamiento quirúrgico por estadio avanzado. Oncología médica en Texcoco la valoró con superficie corporal de 1.83 , y le indicó un ciclo de epirubicina $160 \mathrm{mg}$ trisemanal y de ciclofosfamida $1,100 \mathrm{mg}$ trisemanal, pero la paciente no aceptó la quimioterapia. En su caso, la quimioterapia habría ayudado a disminuir el tumor y a que se volviese operable.

Sin embargo, no acepta la quimioterapia: «por todo lo que dicen que sucede; por todo lo que tiene uno que pasar», así que prefiere una calidad de vida en la cual ella pueda valerse por sí misma.

En este otro caso el miedo se manifiesta como el obstaculizador de la decisión (7). El hecho se presenta «tarde», debido a la posibilidad del diagnóstico tardío. De nuevo surge el problema del desconocimiento in vivo del proceso de atención.

Otra estrategia que puede aplicarse a este caso es el de la atención domiciliaria. El paciente a menudo presenta temor a sufrir fuera de su entorno. Plantear la opción de la atención domiciliaria en caso de agravamiento, lo que implicaría no alejarla de su zona de confort, puede ser un factor para la reducción del rechazo.

Este segundo caso puede entenderse e interpretarse mejor con las etapas clásicas del duelo, aun cuando el pronóstico no sea la 
muerte. Es decir, se presentan las etapas de choque, negación, ira/ enfado, negociación, depresión, resignación/aceptación. Las mismas etapas pueden presentarse en los familiares e incluso en el propio personal sanitario. Hay que recordar que no siempre son secuenciales ni causales de modo necesario; es decir, puede generarse más depresión por la ira o mayor aceptación con la negociación, sin convertirse en algo necesario (8). Los trastornos derivados de la información, la depresión y la ansiedad, son indicadores psicológicos: Las fases de duelo son independientes del tiempo transcurrido desde el diagnóstico; más que fases que definen una secuencia de evolución del duelo se observan respuestas psicológicas al duelo por la pérdida de la salud que coexisten y se interrelacionan (8).

De cualquier forma, requiere un acompañamiento. En un estudio con pacientes de cáncer de mama no se descubrieron diferencias estadísticamente significativas entre los pacientes con cánceres más avanzados en cuanto a la respuesta al duelo (7). Esto nos da una pauta para entender que todos estos síntomas pueden presentarse subjetivamente, sin haber un correlato objetivo de la misma proporción.

Los casos nos muestran que la racionalidad subyacente a ambos casos es la calidad de vida. Hay una percepción de riesgo y de acción desproporcionada médicamente. En ambos, no obstante la intervención médica, auxiliaría objetivamente a mejorar su vida. Entonces, las preguntas que surgen son: ¿Qué limites tiene el consentimiento informado, sin caer en el paternalismo? ¿El paciente verdaderamente comprende las implicaciones y consecuencias de no aceptar el tratamiento? ¿Realmente se comprende que detrás de la negativa del paciente a tratarse subyace un estrés de tal grado, que le impide tomar una decisión acerca de su salud? $(\mathrm{H})$

Lo que proponemos aquí es que se realice una valoración de las distintas variables, para mejorar así la respuesta de los pacientes en el proceso del consentimiento informado. Los estudios han encontrado factores que impiden o, al menos, disminuyen la capacidad 
de decisión del paciente. No hay que olvidar que parte del proceso del consentimiento informado implica ser evaluado en todo el proceso, aunque se haya obtenido la firma legal del mismo.

De hecho, es importante que se respeten las elecciones de la persona enferma, aunque sepamos que hay circunstancias especiales que rodean esta situación y que deben ser discutidas. Los profesionales de salud deben considerar si la información transmitida fue adecuada y si el consentimiento para los tratamientos, cuidados $\mathrm{u}$ otros procedimientos fue libre e informado (7).

En resumen, se han señalado así los conceptos clave de mejora en el proceso de atención del paciente oncológico:

a) Confianza/comunicación real y efectiva.

b) Evitar las sanciones, necesidad de información discutida y analizada junto con el paciente.

c) Considerar siempre el nivel cultural y social del paciente.

d) Tener presente el nivel de verdad tolerable para el paciente, sin mentir.

e) Nunca olvidar la promoción de las virtudes en el paciente y en el personal de salud.

f) Considerar el cuidado del paciente como prioridad, sin descuidar el propio del personal de salud.

g) Combatir el miedo con la familiarización de todo el proceso que debe pasar el paciente.

h) Considerar las etapas de duelo y el distrés presentes en toda decisión del paciente.

Con esos elementos se presenta la propuesta del algoritmo de atención en la primera visita. Se señala el proceso, con las indicaciones en los distintos momentos en los que las categorías deben considerarse y, en paralelo con las funciones de enfermería, con la indicación del patrón funcional de Gordon (9) a recordar en la recopilación de datos para el Plan de Atención de Enfermería (PAE): 


\section{J. E. Gómez Álvarez, N. H. Chávez Hernández}

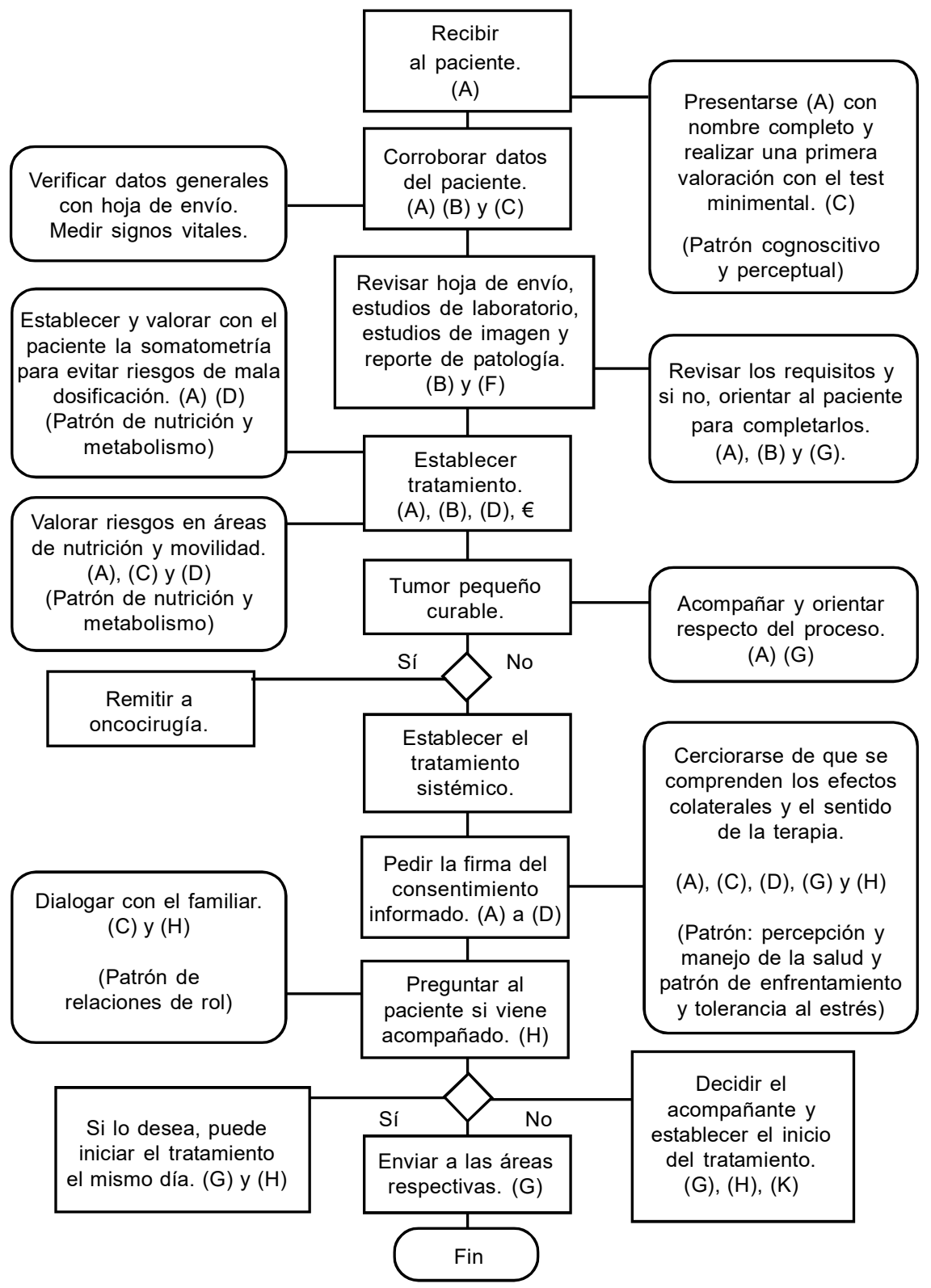

Figura 1. Algoritmo decisional en el proceso de atención de un paciente oncológico.

Fuente: Elaboración propia. 
Análisis del consentimiento informado en pacientes con cáncer...

En el mismo se han remarcado momentos esenciales en los que se debe cuidar al máximo la capacidad virtuosa y prudencial del proceso de atención. Valorar riesgos de nutrición y movilidad implica no subestimar ni sobreestimar las capacidades del paciente para autorregularse; es decir, la templanza. Debe ser examinada no la mera recomendación o proporcionar una guía, sino comprender las dificultades del paciente y vigilar el modo de proceder del prestador de servicios de salud. Éste ha de tener la justa medida entre conmiseración e indiferencia, y actuar de acuerdo con el justo medio respecto de la situación.

\section{Conclusiones}

Podemos suscribir lo expresado por Allende S. et al: El CI no es un simple requisito legal o una autoprotección del médico o de los hospitales contra una demanda, sino que es el ritual y proceso informativo que todo profesional ejerce con responsabilidad para cuidar la atención paliativa (2). Aunque hace referencia a los cuidados paliativos, esto es extensible al primer contacto del paciente que todavía es tratable. Tanto el médico como el enfermero deben recopilar información para establecer pautas de intervención con los pacientes, de modo que ambos realicen una labor sinérgica que lleve a la obtención de un auténtico consentimiento informado, mediante la recopilación de datos que garantice una atención del médico y del enfermero, de manera que permita perdurar más allá de la firma del documento a lo largo de todo el proceso de atención.

\section{Notas bibliográficas}

1 Ubicada en Av. Solidaridad s/n, Col. Providencia, Valle de Chalco, Edo. de México.

${ }^{2}$ La mejora se expresa por medio de un algoritmo de decisión, en donde se van señalando los conceptos a considerar. En el texto, se indican con letras los térmi- 
nos a considerar y, en el algoritmo, se señala el o los momentos en los que deben considerarse los mismos.

${ }^{3}$ El test minimental es un cuestionario breve utilizado sobre todo en pacientes geriátricos, para medir si hay presencia de deterioro cognitivo. Consta de 30 ítems. Puede verse el mismo en: https://www.farmaceuticoscomunitarios.org/anexos/vol 11_n1/ANEXO2.pdf

\section{Referencias bibliográficas}

1. Martínez P, Andreu Y, Galdón M, Romero R, García A, Llombart P. Distrés emocional y problemas asociados en población oncológica adulta. Psicooncología. 2017; 14(2-3): 217-228. [Consultado en febrero de 2019]. Disponible en: https:// www.researchgate.net/publication/320385362_Distres_emocional_y_problemas_ asociados_en_poblacion_oncologica_adulta https://doi.org/10.5209/psic.57081

2. Allende S, Verástegui E, Mohar A, Meneses A, Herrera S. La pieza perdida en la medicina actual: atención domiciliaria a pacientes paliativos oncológicos. Salud Pública Méx. 2016; 58: 229-233. [Consultado en febrero de 2019]. Disponible en: http://www.scielo.org.mx/pdf/spm/v58n2/0036-3634-spm-58-02-00317.pdf

3. Verástegui E, Allende S. Aspectos éticos en pacientes con cáncer avanzado en cuidados paliativos. Gaceta Mexicana de Oncología. 2013; 12 (4): 250-255.

4. Ataupillco Y, Sánchez O, Saravia F. Efectividad de un programa de consejería de enfermería en las estrategias de afrontamiento en mujeres con cáncer de mama (Tesis de especialidad). Lima, Perú: Universidad peruana Cayetano Heredia; 2016. [Consultado en febrero de 2019]. Disponible en: https://alicia.concytec.gob.pe/vufind/Record/RPCH_Of86aa40c283d51419f524376bf5357d/Details

5. Rubiales A, Del Valle M, García C, Garavís M, Vecino A, Hernansanz H, López F. Información al enfermo oncológico: los límites de la «verdad tolerable». Cuad Bioet. 1998; (1): 388-401. [Consultado en febrero de 2019]. Disponible en: http:// aebioetica.org/revistas/2000/3-4/43/388.pdf

6. Vigo A. Ética general. DuocUC. Centro de Ética Aplicada. Pontificia Universidad Católica de Chile. S. f. [Consultado en noviembre de 2020]. Disponible en: https:// www.academia.edu/35224318/Etica_General_Rodolfo_Vigo

7. Valderrama M, Sánchez R. Trastornos de ansiedad y depresión en relación con la calidad de vida de pacientes con cáncer de mama en estadio localmente avanzado o diseminado. Rev Colomb Psiquiat. 2017; 47(4): 211-220. [Consultado en febrero de 2019]. Disponible en: http://www.scielo.org.co/scielo.php?script=sci_abstract\&pid=S0034-74502018000400211\&Ing=en\&nrm=iso https://doi.org/10. 1016/j.rcp.2017.04.003

8. Moral J, Melina M. Pensamiento psicológico. 2014; 13 (1): 7-25. [Consultado en febrero de 2019]. Disponible en: http://www.scielo.org.co/pdf/pepsi/v13n1/v13n1a01.pdf 
Análisis del consentimiento informado en pacientes con cáncer...

9. Cisneros F. Proceso de atención de enfermería. Universidad de Cauca. [Consultado en febrero de 2019]. Disponible en: http://artemisa.unicauca.edu.co/ pivalencia/archivos/ProcesoDeAtencionDeEnfermeria-PAE.pdf 
